\title{
Safe Handling of TBP and Nitrates in the Nuclear Process Industry (U)
}

by

M. L. Hyder

Westinghouse Savannah River Company

Savannah River Site

Aiken, South Carolina 29808

This paper was prepared in connection with work done under the above contract number with the U.S. Department of Energy. By acceptance of this paper, the publisher and/or recipient acknowledges the U.S. Government's right to retain a nonexclusive, royalty-free license in and to any copyright covering this paper, along with the right to reproduce and to authorize others to reproduce all or part of the copyrighted paper. 


\section{DISCLAIMER}

This report was prepared as an account of work sponsored by an agency of the United States Government. Neither the United States Government nor any agency thereof, nor any of their employees, make any warranty, express or implied, or assumes any legal liability or responsibility for the accuracy, completeness, or usefulness of any information, apparatus, product, or process disclosed, or represents that its use would not infringe privately owned rights. Reference herein to any specific commercial product, process, or service by trade name, trademark, manufacturer, or otherwise does not necessarily constitute or imply its endorsement, recommendation, or favoring by the United States Government or any agency thereof. The views and opinions of authors expressed herein do not necessarily state or reflect those of the United States Government or any agency thereof. 


\section{DISCLAIMER}

Portions of this document may be illegible in electronic image products. Images are produced from the best available original document. 
Key Words: ITP

Nitric Acid

PUREX

Solvent Extraction

Chemical Reaction

Retention: Lifetime

\section{Safe Handling of TBP and Nitrates in the Nuclear Process Industry (U)}

Author:

M. L. Hyder

July, 1994

Westinghouse Savannah River Company Savannah River Technology Center Aiken, SC 29808

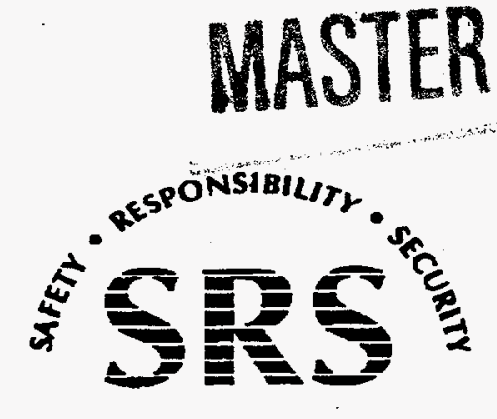

PREPARED FOR THE U.S. DEPARTMENT OF ENERGY UNDER CONTRACT NO. DE-AC09-89SR18035 
WSRC-TR-94-0372

Rev. 0

Project:

Document:

Title:

Approvals:

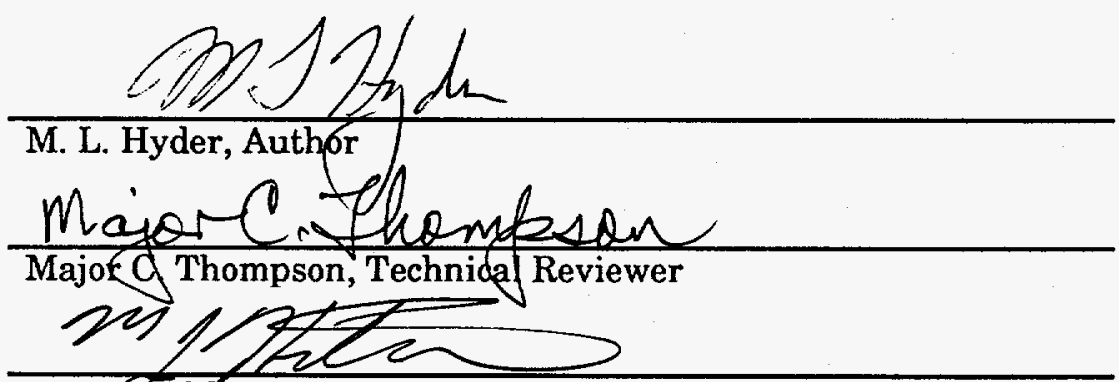

M. J. Hitchler, Manager, Safety Analysis Engineering
Tomsk Technical Studies

WSRC-TR-940372

Safe Handling of TBP and Nitrates in the Nuclear Process Industry (U) 


\section{TABLE OF CONTENT}

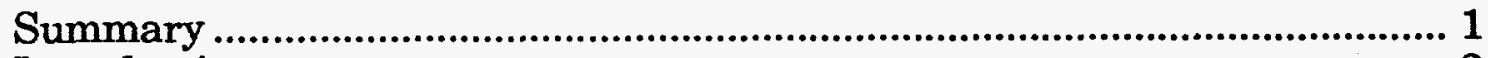

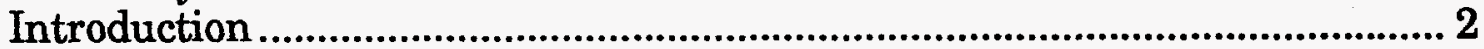

Energetics ............................................................................................. 7

Heat Transfer and Venting .................................................................... 8

Conclusions and Recommendations ......................................................... 10

References........................................................................................ 12

Figure 1: TBP Acid Hydrolysis \& Reaction Rates ........................................ 14

Figure 2: Butene Production vs. Temperature ............................................. 15

Figure 3: Effect of Vent Size on Internal Pressure ..................................... 16 
WSRC-TR-94-0372

Rev. 0

Page 1 of 18

\section{Summary}

At the request of the USDOE Defense Programs Office of Special Projects, a laboratory and literature study was made of the reactions of tri-n-butyl phosphate (TBP) with nitric acid and nitrates. Its goal was to establish safe conditions for solvent extraction processes involving these chemicals. The damaging explosion at the Tomsk-7 PUREX plant in Russia graphically illustrated the potential hazard involved in such operations. The study has involved a review of prior and contemporary experiments, and new experiments to answer particular questions about these reactions.

TBP extracts nitric acid and some metal nitrates from aqueous solutions. The resulting liquid contains both oxidant and reductant, and can react exothermically if heated sufficiently. (Reaction is negligibly slow at the temperatures used for solvent extraction.) Reaction products include flammable or reactive gases. In addition to the Tomsk 7 explosion, at least four incidents of damage in North American plants have been attributed to such reactions since 1953.

Safe handling of these potentially reactive materials involves not only limiting the heat generated by the chemical reaction, but also providing adequate heat removal and venting. Effective heat removal can stabilize the solution temperature and reaction rate; venting can prevent the pressure buildup that leads to explosions. Specifically, the following recommendations are made to ensure safety:

(1) Tanks in which TBP-nitrate complexes are or may be present should be adequately vented to avoid pressurization. Data are supplied as a basis for adequacy.

(2) Chemically degraded TBP, or TBP that has sat a long time in the presence of acids or radiation, should be purified before use in solvent extraction. Alcohols and other compounds in degraded TBP can react exothermically with nitric acid, and these need to be removed before the TBP is heated or exposed to strong acid.

(3) Evaporators in which TBP might be introduced should be operated at a controlled temperature, and their TBP content should be limited.

(4) Evaporator bottoms that may contain TBP should be cooled under conditions that ensure heat removal.

Finally, process design should consider the potential for such reactions, and operators should be made aware of this potential, so that it is considered during training and process operation. 


\section{Introduction}

The study reported here was undertaken at the request of the USDOE Defense Programs Office of Special Projects, following the damaging explosion at the Tomsk7 nuclear fuel reprocessing plant in Russia during April, 1993. ${ }^{1}$ Its goal was to establish safe conditions for the handling of nitric acid and tri-n-butyl phosphate (TBP) during PUREX and related chemical processing. Although these reagents are mutually compatible at normal PUREX operating temperatures, there is a potential for dangerously rapid oxidation of TBP at higher temperatures. In addition to the Tomsk-7 explosion, at least four incidents in North American plants have been attributed to such reactions. ${ }^{2-5}$

The explosion in the Tomsk-7 plant involved reaction of strong (14M) nitric acid with organic material originating from a PUREX type of solvent extraction process. The organic material was not well characterized, but presumably contained TBP and its degradation products. The materials were contacted in a tank that also contained evaporator bottoms (probably still thermally hot). Venting was absent or restricted until a substantial pressure, about $3 \mathrm{~atm}$., had been generated in the vessel. 17 During a period of about 100 minutes, an accelerating reaction occurred that overwhelmed the pressure relief and finally burst the vessel. Substantial damage to the building was done by the resulting pressure wave and/or ignition of flammable gases released from the vessel.

It had long been known that nitric acid and nitrates can oxidize organic material at high temperatures, and this has been exploited in a number of industrial processes. Nitration of TBP was recognized as a potential concern to the nuclear process industry in 1953, after damaging incidents at Hanford and Savannah River. 2,5 In each case TBP solution was inadvertently allowed to enter an evaporator in which a nitrate solution was being concentrated at a relatively high temperature. (The temperature at Savannah River was not controlled or recorded.) The damage at Hanford was minor, but the unit at Savannah River was destroyed by an explosion. Temperature controls were established by the two sites following investigations of these incidents, and these have successfully prevented any recurrence in evaporators within the DOE complex. However, an incident that damaged a Canadian evaporator in 1980 appears to have been caused by a nitrate-TBP reaction. ${ }^{4}$ This incident, which occurred in the second stage of a two-stage evaporation process, may have involved conditions rather similar to the other evaporator incidents. Some reports suggest that one or more additional incidents may also have occurred in Soviet Union plants, but details are not available. Ref 5a

A damaging incident at Savannah River in 1975 resulted from the heating of TBP in a calciner vessel with uranyl nitrate. ${ }^{3}$ In this case the calciner was adequately vented, but the fumes released to the process room were flammable and ignited, producing a fireball and a pressure wave that blew out lightly constructed walls. It is of interest that the TBP, which unintentionally entered the calciner, had already 
safely passed through an evaporator. This supports the effectiveness of the temperature controls for such evaporators.

These incidents led to investigations that produced most of the information previously available on the TBP-nitrate reaction. Wagner performed a number of early qualitative studies at Hanford. ${ }^{7}$ At Savannah River, Nichols conducted isothermal tests in sealed tubes that established the kinetics of nitrate-TBP reactions in the organic phase, in addition to providing information on the reaction products and energetics. ${ }^{8}$ Following the 1975 incident, Harmon et al. conducted thermal analyses on the TBP-uranyl nitrate adduct, establishing that the principal flammable gas evolved from heating this adduct is 1-butene. 9 This is produced by pyrolysis reactions at temperatures above $130^{\circ} \mathrm{C}$. This study also provided information on the oxidized products and the kinetics of the various reactions. Subsequently, in the course of modifications to this calciner facility, additional studies were conducted by Gordon. 10,11 His experiments involved heating TBP with nitric acid or nitrate solutions in a sealed pressure vessel. At high temperatures $\left(160^{\circ}-200^{\circ} \mathrm{C}\right)$ and pres $2 u r e s$ of several atmospheres abrupt reactions were observed that had many of the characteristics of ignitions. Earlier, and subsequent, studies in open vessels had not observed pressure surges of this type, and a reasonable hypothesis is that they represent a gas-phase "burn" of butene and CO by nitrogen oxides, spontaneously initiated at the high temperature and pressure inside the vessel. The pressure conditions at Tomsk-7 were similar to those studied by Gordon, exceeding the gauge limit of $6 \mathrm{~atm}$. before the rupture occurred. Head space temperatures may also have been fairly high. Thus it is plausible that such a burn may have occurred there. It would have provided the pressure surge leading to the sudden vessel rupture. Thompson and Cavin at Savannah River have observed an (externally ignited) burn during heating experiments in open vessels, which lends credibility to this hypothesis. ${ }^{12}$

Studies of the nitrate-TBP reaction have also been conducted in Russia. 13 These have noted that significant amounts of carbon monoxide can be produced in the reaction. (Russian investigators tend to attribute much of the damage at Tomsk-7 to ignition of released CO.)

Before the Tomsk-7 explosion, all known incidents involving TBP-nitrate reactions had involved an external heat source. The Tomsk-7 incident ostensibly did not, although a portion of the vessel contents was fresh from the evaporator and therefore still hot. (Some reports suggest that steam injection into the vessel may have provided additional heating. ${ }^{14}$ ) Some additional heating may have been provided through dilution of the concentrated nitric acid. However, any such heating of the solution only provided the conditions under which exothermic chemical reactions became significant. Subsequent heating was caused by the chemical heat from these reactions. These are believed to have occurred in the organic phase, which was floating on the aqueous phase. Downward heat transfer is slow, because it does not generate convection; thus the organic phase may have heated to a temperature much higher than the aqueous phase. In addition, the 
Tomsk vessel vent may have initially been closed, so that evaporative cooling was ineffective. There was therefore little cooling to counteract the chemical heating.

Nichols had suggested as early as 1960 that a TBP-nitric acid mixture floating on a hot solution in a large vessel could self-heat, beginning in some cases with temperatures of $100^{\circ} \mathrm{C}$ or less. 8 He presented curves based on assumed heat transfer values for calculating the heat balance temperature in cylindrical vessels. The data were developed as a function of vessel size and organic content. (In fact, because of errors in his thermodynamic data, his curves underestimate the potential heat production.) Although Nichols' report was circulated within DOE and subsequently made available to the public, the possibility of self-generated heating does not seem to have been considered in U.S. safety analyses until recently. The Tomsk-7 incident apparently has substantiated Nichols' hypothesis.

Thus for establishing safe operating conditions it is not enough to consider the generation of heat and gases by nitrate-TBP reactions; heat removal and ventilation processes must be incorporated into the overall evaluation. These considerations are developed here in more detail, to obtain guidelines for safe handling of TBP/nitrate mixtures.

Chemistry and Kinetics of the TBP-Nitrate Reaction

All the chemical and kinetic considerations discussed here involve the organic phase only; the low solubility of TBP in the aqueous phase makes aqueous phase reactions of much less concern. (A possible exception is the case of degraded TBP, as will be discussed.) The aqueous phase is involved primarily as the source of the nitric acid (and of water) which is taken up by the TBP. Equilibrium concentrations of nitric acid and water in pure TBP contacted with aqueous nitric acid have been published by Davis et al. ${ }^{15}$ Representative data are shown in Table 1. As indicated there, over the ranges of acidity encountered in process solutions, the ratio of nitric acid to TBP ranges up to about 1.5. In solutions containing uranyl nitrate, the ratio of nitrate to TBP is typically about 1 . These quantities of nitrate are sufficient to oxidize less than 10 per cent of the TBP present, so complete reaction leaves most of the TBP unaffected. However, if the reaction continues long enough, additional acid may be extracted from the aqueous phase to replace that lost by reaction or evaporation. Additionally, the heat generated and the accompanying temperature rise may cause additional thermal decomposition of the TBP. Consequently, a larger part of the TBP may be involved in reactions. 
WSRC-TR-94-0372

Rev. 0

Page 5 of 18

Table 1

Composition of TBP Equilibrated With Aqueous Nitric Acid (From Ref. 15)

Aqueous Phase

$\underline{\mathrm{HNO}}_{3}, \mathrm{M}$

2.0

4.0

6.0

8.0

10.0

12.0

14.0
Organic Phase

$\mathrm{HNO}_{3}, \mathrm{M}$

$\underline{\mathrm{H}_{2}} \underline{\underline{O} . \mathrm{M}}$

TBP, M

1.48

2.33

2.92

3.37

3.82

4.37

5.79
2.76

1.95

1.40

1.20

1.24

1.41

2.00
3.26

3.17

3.11

3.04

2.97

2.87

TBP as used in solvent extraction is diluted with a solvent that enables rapid and complete separation of the two phases. Usually this is a light organic solvent that decreases the density of the TBP phase, but carbon tetrachloride has also been used for this purpose; in this case the organic phase is heavier than the aqueous phase. It has been suggested that reactions of organic diluent with nitric acid may contribute to the overall reaction. Diluents containing aromatic compounds, or even branched aliphatic compounds may react with nitric acid when heated, and such reactions are believed to give the red color in "red oils". A recent Los Alamos study has explored this question. ${ }^{6}$ However, the diluents currently used in the U.S. generally contain few such reactive components; they are mostly straight chain hydrocarbons. Additionally, all the incidents that took place in the U.S. occurred under circumstances in which any diluent originally present probably had been removed by distillation. In general, then, the diluent is probably a positive factor. As long as it is present, it dilutes the nitric acid and TBP in the organic phase, thereby reducing the heating and reaction rates. The situation at Tomsk-7 is unclear. The diluent used included some aromatics and branched hydrocarbons, 17 but it is uncertain how much diluent, if any, was present at the time of the incident.

The sealed tube studies of Nichols characterized the kinetics of reaction of TBP/nitric acid and TBP/nitric acid-uranyl nitrate systems as a function of temperature. 8 The technique used was to measure the product gases accumulated in isothermal sealed tubes as a function of time. Nichols showed that, at constant temperature, the reaction rate could be fitted to a first order decay curve of the type 
WSRC-TR-94-0372

Rev. 0

Page 6 of 18

$\mathrm{dN} / \mathrm{dt}=-\mathrm{kN}$

where $\mathrm{N}$ is a substance that is reacting to produce the observed gases. Inasmuch as there is a large excess of TBP over nitric acid or nitrate in these organic solutions, $N$ can be identified with nitrate. The dependence of the rate constant $k$ on temperature was shown to follow the Arrhenius relationship:

$\mathrm{k}=\mathrm{se} \mathrm{e}^{(\mathrm{RT} / \mathrm{E})}$

where $s$ is the frequency factor for the reaction, $R$ the gas constant, $T$ the absolute temperature, and $E$ the activation energy. The activation energy $E$ for the reaction was constant for all the conditions investigated, but the frequency factor $\mathrm{s}$ varies with the nitric acid and uranyl nitrate concentrations in the organic phase. This factor $s$ is not a strong function of the nitric acid concentration with which the TBP was originally equilibrated. However, addition of uranyl nitrate to the organic solution decreases the value of $s$, i. e., slows the reaction.

Using the Arrhenius parameters, the reaction rate can be extrapolated to lower temperatures where it is not directly measurable. Such an extrapolation is shown in Figure 1. At these lower temperatures the rate of acid-catalyzed hydrolysis of TBP has been measured, and the results are compared to the extrapolated reaction rate data for TBP-nitric acid in Figure 1. The congruence of the curves suggest the hydrolysis of TBP is the initial step in the reaction process, or that the hydrolysis and the nitration reaction share a common first step. (The bond breakage leading to reaction is reported to involve the $\mathrm{C}-\mathrm{O}$ bond.) ${ }^{16}$ It has been reported that butyl nitrate is produced instead of butanol at nitric acid concentrations of $2 \mathrm{M}$ or greater. 18,19 However, recent measurements by Smith indicate that both compounds are produced. ${ }^{20}$

A second reaction, first characterized by Harmon et al., 9 and more recently by Barney and Cooper, 21 is the pyrolysis of TBP at temperatures of $120^{\circ} \mathrm{C}$ or above. The study by the latter authors has determined the temperature dependence of this reaction, which is not as steep as that of the hydrolysis or nitration. Their results, based on butene measurements, are summarized in Table 2 and Figure 2 . The temperature dependence of this reaction is less rapid than the competing oxidation reaction. Consequently the relative rate of pyrolysis decreases with temperature until the oxidation reaction is complete. At higher temperatures the nitric acid is mostly reacted and/or evaporated, and the pyrolysis reaction dominates. 
Table 2

\section{Butene Evolved from Heated TBP (From Ref. 21)}

Temperature, ${ }^{\circ} \mathrm{C}$

112

132

152
First Order Rate Constant, sec-1

$1.90 \times 10^{-5}$

$1.11 \times 10^{-4}$

$2.61 \times 10^{-4}$

TBP can be degraded over time by radiation and/or by acidic or basic hydrolysis. The result is the loss of a butyl group, forming dibutyl phosphate (DBP) and either butanol or butyl nitrate. DBP is more water soluble than TBP, and can precipitate plutonium and other metals. Both butanol and butyl nitrate react exothermically with nitric acid at elevated temperatures, and their accumulation could provide a heating mechanism when the degraded solvent is contacted with hot nitric acid. In the case of butanol, which partially strips into the aqueous phase, reaction with nitric acid occurs above $60^{\circ} \mathrm{C}$. This can provide a quick increase in the aqueous temperature. ${ }^{12}$ Butyl nitrate can play a similar role in the organic phase. In SRS PUREX operations, the solvent is regularly washed successively with dilute alkali and acid to remove impurities, and this washed solvent is relatively free of impurities. Solvent stored for a long time without washing is more hazardous; this may have been an important factor in the Tomsk-7 incident. In this case, the majority of the organic material had been stored for a long time, perhaps several years, in contact with an acidic solution. (The original report of storage time was "at least six months"; subsequent discussion revealed that it may have been considerably longer. The organic material involved included material that accumulated at the pulse column interface during processing and thus already had undergone some degradation.)

An important effect in the overall energy balance is physical, rather than chemical. This is the evaporation of dissolved water and nitric acid from the organic phase when heated. Inasmuch as water is present at $1 \mathrm{M}$ or more and nitric acid may be present at concentrations $>3 \mathrm{M}$, this can be a significant effect. The studies by Barney and Cooper ${ }^{21}$ and by Smith ${ }^{20}$ have shown that this effect can dominate the energy balance for some time (many minutes) in TBP at temperatures below $120^{\circ} \mathrm{C}$ or so, causing a net cooling and slowing the chemical reaction. It also removes a portion of the nitric acid reagent. In their DTA experiments Barney and Cooper showed a substantial reduction in the net energy of reaction, a part of which is the result of this evaporative cooling effect. This is further discussed below. 
WSRC-TR-94-0372

Rev. 0

Page 8 of 18

\section{Energetics}

It is assumed in the following discussions of reaction energetics that the reactions of nitrate and TBP presented adequately describe the behavior of the TBP-nitrate system at high temperature. It is further assumed that diluent and reactive products of diluent nitration are absent. Energetics are developed in terms of the complete oxidation of TBP. Actually, decomposition and oxidation will proceed stepwise, through the di- and mono-butyl phosphates, which are observed as products. ${ }^{12}$ This will have some effect on the energetics, but probably a rather minor one, as hydrolysis of TBP is not very energetic. The overall considerations should not be affected by this.

Complete oxidation of TBP to water, carbon dioxide, and phosphoric acid through the reaction

$$
\mathrm{OP}\left(\mathrm{OC}_{4} \mathrm{H}_{9}\right)_{3}+14.4 \mathrm{HNO}_{3} \Rightarrow=>\mathrm{H}_{3} \mathrm{PO}_{4}+7.2 \mathrm{~N}_{2}+12 \mathrm{CO}_{2}+19.2 \mathrm{H}_{2} \mathrm{O}
$$

generates heat $\left(\Delta \mathrm{H}^{\circ}\right)$ of about $1540 \mathrm{kcal} / \mathrm{mole} \mathrm{TBP}$, or $107 \mathrm{kcal} / \mathrm{mole}$ nitric acid. The extent of reaction is limited by the concentration of nitric acid in the organic phase, which is usually not much more than that of the TBP. Consequently, less than $10 \%$ of the oxidant required for complete oxidation of TBP is available. Additionally, if the reaction does not go to completion as written, but produces intermediate products that evaporate, the energy is further reduced. Some examples of reaction energies for a number of possible reactions are given in Table 3. Nichols' sealed tube experiments determined some of the products, and he used these measurements for the calculation of the energy required to produce them. When corrected for $\mathrm{CO}$ formation, and for an assumed yield of water, the energy released by complete reaction of the nitric acid present is calculated to be about $340 \mathrm{kcal} / \mathrm{kg}$ (cal/g) for TBP equilibrated with strong (up to $10.7 \mathrm{M}$ ) nitric acid. ${ }^{22}$

In situations that permit gases to be easily evaporated and removed from the vapor phase, the energy release is substantially lower. Barney and Cooper 21 made measurements in a differential scanning calorimetry cell and obtained exotherms corresponding to $100-140 \mathrm{cal} / \mathrm{g}$. These measurements were obtained by integrating the reaction exotherm, which was adjacent to the endothermic evaporation peak. It is possible that the two may have canceled one another to some extent.

Calculations from the reaction product data obtained by Harmon, et. al, ${ }^{9}$ with a similar apparatus, give a higher value: 200 to $225 \mathrm{cal} / \mathrm{g} .{ }^{23}$ This would seem to be a reasonable conservative value for a vented system, in that it is largely based on gas yields, and should not be complicated by endothermic processes such as evaporation. Again, the calculation is based on complete reaction of the nitric acid in the organic phase. 


\section{Table 3}

\section{Reactions and Energies}

$\mathrm{OP}\left(\mathrm{OC}_{4} \mathrm{H}_{9}\right)_{3}+24 \mathrm{HNO}_{3}=\Rightarrow \mathrm{H}_{3} \mathrm{PO}_{4}+24 \mathrm{NO}+12 \mathrm{CO}_{2}+24 \mathrm{H}_{2} \mathrm{O}$

$\Delta \mathrm{H}^{\circ}=-821 \mathrm{kcal} / \mathrm{mole} \mathrm{TBP},-34 \mathrm{kcal} / \mathrm{mole}$ nitric acid

$\mathrm{OP}\left(\mathrm{OC}_{4} \mathrm{H}_{9}\right)_{3}+16 \mathrm{HNO}_{3} \Rightarrow \mathrm{H}_{3} \mathrm{PO}_{4}+16 \mathrm{NO}+12 \mathrm{CO}+20 \mathrm{H}_{2} \mathrm{O}$

$\Delta \mathrm{H}^{\circ}=-425 \mathrm{kcal} / \mathrm{mole} \mathrm{TBP},-26 \mathrm{kcal} / \mathrm{mole}$ nitric acid

$\mathrm{OP}\left(\mathrm{OC}_{4} \mathrm{H}_{9}\right)_{3}+18 \mathrm{HNO}_{3}=\Rightarrow \mathrm{H}_{3} \mathrm{PO}_{4}+9 \mathrm{~N}_{2} \mathrm{O}+12 \mathrm{CO}_{2}+21 \mathrm{H}_{2} \mathrm{O}$

$\Delta \mathrm{H}^{\circ}=-1287 \mathrm{kcal} / \mathrm{mole} \mathrm{TBP},-72 \mathrm{kcal} / \mathrm{mole}$ nitric acid

Recent experiments by Smith ${ }^{20}$ at Savannah River have emphasized the importance of evaporative processes in lowering the available energy. This involves both loss of the nitric acid oxidant by evaporation, and the uptake of heat by the endothermic evaporation process. In one experiment, in which TBP was equilibrated with $10 \mathrm{M}$ nitric acid and held at $125^{\circ} \mathrm{C}$ for 6.5 hours, nearly half the nitric acid was distilled as nitric acid or recovered as unreacted butyl nitrate. Most of that which reacted produced $\mathrm{NO}$ or $\mathrm{N}_{2} \mathrm{O}$. Consequently, the net energy released was greatly reduced. The total chemical energy was calculated from the heats of formation of the reaction products to be less than $100 \mathrm{cal} / \mathrm{g}$. Heat taken up by evaporation would have reduced this still further.

Experimentally, self-heating of TBP containing nitric acid is generally not observed in laboratory equipment at temperatures below $125^{\circ} \mathrm{C}$, but it is generally observed, even in vessels open to the atmosphere, by the time $135^{\circ} \mathrm{C}$ is reached. $8,11,12$ These findings were used at the Savannah River Site to establish a limit of $130^{\circ} \mathrm{C}$ on the steam used to heat evaporators.

\section{Heat Transfer and Venting}

Once a quantity of TBP-nitrate is heated to the point that it begins to react at an appreciable rate, the course of subsequent reactions is determined by the heat removal processes available. These may include:

Evaporation of water or other materials;

Heat transfer by conduction or convection to an aqueous phase;

Heat transfer to the vessel walls;

Convective cooling by the gas in the head space;

Cooling by installed cooling coils. 
The effect of evaporation is extremely important, as has been shown by Smith. 20 TBP in equilibrium with aqueous solutions contains enough water to take up all the chemical heat of reaction for periods of the order of a half hour at $110^{\circ} \mathrm{C}$.

Furthermore, for TBP in contact with an underlying water layer, additional water can be taken up or vaporized from this layer. Therefore, in apparatus in which evaporation is possible, a substantial margin of safety is provided against runaway reactions. This is a major reason why such reactions are not observed in open vessels until temperatures in excess of $130^{\circ} \mathrm{C}$ are reached.

In an unstirred enclosed vessel, the geometric aspects are very important for determining the available heat transfer mechanisms. Chemical heating is occurring in an organic phase overlying an aqueous phase. The floating organic phase is in the form of a disc, with its radius being much greater than its thickness. Organic solutions are poor conductors of heat. Consequently, only a small region of the organic phase can be affected by cooling at the vessel walls, and the geometry will prevent establishing large-scale convection processes within the organic phase. The top of the aqueous phase is being heated, producing a less dense top layer.

Consequently, there are no gravitational forces producing convection in this phase. Downward heat transfer between the phases therefore involves conduction, rather than convection, and heat removal is consequently slower. In such systems, therefore, cooling occurs mainly by evaporation or by convective cooling by the air entering the head space. Both processes are facilitated by circulating unsaturated air through the head space, which was not done at Tomsk-7.

In well-mixed vessels that are sufficiently vented, evaporation of water can readily maintain a constant temperature. In an evaporator, for example, in which the entire contents are being heated, the resultant turbulent mixing should keep the organic and aqueous phases at the same temperature, so that the overall temperature is controlled by the boiling of water. In any well-mixed and sufficiently vented vessel containing aqueous and organic phases, the two phases should remain in thermal equilibrium, and heat removal by boiling will occur if the boiling point of either phase is attained. This should prevent the occurrence of a runaway reaction. Either air sparging or mechanical mixing could accomplish this.

Even in vessels that are not well mixed, experiments have shown that venting the TBP-nitric acid reaction can prevent pressurization and a runaway reaction by permitting evaporation and the release of product gases. Fauske conducted these experiments using apparatus developed for sizing safety vents in chemical plants. ${ }^{24}$ This apparatus maintains the surroundings of a small reaction vessel isothermal with the vessel, so that the internal reaction is not affected by heat transfer through the vessel. Some of the resulting data are shown in Figure 3. These data indicate that a vent of at least $0.032 \mathrm{~cm}^{2} / \mathrm{kg}$ organic is required to limit pressurization of a nitric acid-TBP system. (See Figure 3)

The mass/vent ratio at Tomsk-7 is also shown in Figure 3, based on a $7 \mathrm{~cm}$ vent line open to $70 \%$ of its diameter, and estimates of 200 to 500 liters of organic material present. The figure shows that the larger volumes would have been within the 
unsafe region. Additionally, venting did not become fully effective until a pressure of $3 \mathrm{~atm}$. had already been reached. 17 This prevented effective evaporative cooling, and allowed the reaction to progress further than it would have with adequate venting. SRS experiments have confirmed that allowing the pressure to build up above atmospheric pressure markedly increases the venting requirement. ${ }^{12}$

Another means of cooling the organic phase would be the use of cooling coils, if the cooling surface is in contact with the organic phase. Cooling coils extending throughout the aqueous phase would also promote convective cooling on the lower surface of the organic phase.

Finally, chilling the head space of the vessel would provide a mechanism for cooling the surface of the enclosed liquid by air convection and by evaporation. As an example, the condenser of an evaporator could be operated during the cool-down of the evaporator bottoms, as proposed for the British THORP plant. 24

\section{Conclusions and Recominendations}

All the serious accidents resulting from TBP-nitrate reaction in the nuclear industry have been at least in part the result of TBP being placed in an apparatus where it is not normally expected. Although considerable efforts may be made to separate TBP from the aqueous phase in solvent extraction operations, they do not always succeed. Consequently, it is prudent to allow for the possibility of nitrateTBP contact under unplanned circumstances. This is particularly true in operations that involve heating and concentrating nitrate or nitric acid solutions. This does not mean that attention to the control of TBP should be lessened, for it remains the first and most important protection against the introduction of TBP into a potentially dangerous part of the operation.

1. Ventilation of the vessels is the first defense against a damaging runaway reaction. Based on the data obtained by Fauske ${ }^{25}$, in vessels containing nitric acid where TBP accumulation is a concern, a vent should be provided that provides a vent area of $0.032 \mathrm{~cm}^{2}$ per kg of TBP that it is considered possible to accumulate in them. Maintaining continuous air flow through the head space is also desirable, as it provides cooling and removes accumulating gases.

2. TBP that has degraded as the result of radiation or hydrolysis should be washed and tested before reuse. TBP allowed to stand for several months in the presence of aqueous acids or bases and/or radiation should be regarded as suspect in this regard.

3. Evaporators in which TBP may be present should be controlled to prevent local high temperatures. No portion or surface of the evaporator should exceed $130^{\circ} \mathrm{C}$, and operating temperatures substantially lower than this are desirable. If evaporators, calciners, or other heating units must operate at higher temperatures, 
the procedures for preventing the introduction of TBP into them must be correspondingly rigorous.

4. Evaporated solutions are a particular concern, as they come from the evaporator hot, and are typically allowed to settle while cooling. A variety of measures may be appropriate to guard against runaway reactions of TBP unintentionally introduced into such solutions. Sparging, mechanical mixing, cooling by cooling coils, operation of the condenser, or provision of a large vent may be appropriate for this purpose, depending on the design of the system and the perceived hazard.

Finally, it is important that the operators of PUREX and similar solvent extraction processes be aware of the potential for reaction of nitric acid or nitrates with TBP. Perhaps the greatest potential for adding to the list of accidents lies in non-standard operations that must be performed from time to time. This was the origin of the Tomsk-7 accident: inadequate care was taken during a recycle process to make sure that no hazards would be generated. The potential for such accidents should be taken into account whenever changes are made in process or equipment. 
WSRC-TR-94-0372

Rev. 0

Page 13 of 18

\section{References}

1. F. C. Gilbert, F. E. Witmer, R. E. Felt, L. W. Gray, M. L. Hyder, L. H. Sullivan, and D. D. Wodrich, "Trip Report, Moscow and Tomsk, Russia, June 19-29, 1993", DOE/DP-0120, September, 1993.

2. T. J. Colven, Jr., G. M. Nichols, and T. H. Siddall, "Interim Technical Report, TNX Evaporator Incident January 12, 1953", DP-25, May 15, 1953.

3. L. W. Gray, "An Explosion and Fire During Conversion of Liquid Uranyl Nitrate to Solid Uranium Oxide", Nucl. Safety 19, 91 (1978).

4. J. W. Carrigan, A Report on a UNH Boildown Evaporator Explosion at Port Hope's Uranium Refinery Ju? y 16, 1980, Undocumented Eldorado Nuclear Ltd. Report, August 20,1980. Additional information on this incident, some contradictory to the published report, was obtained by A. P. Gouge of WSRC through personal contacts.

5. D. O. Campbell and J. C. Mailen, The Red-Oil Problem and Its Impact on PUREX Safety, ORNL/TM-10798, June, 1988.

6. J. G. Watkin and P. L. Gordon, Investigation of "Red Oil" and its Stability in Simulated Hanford Tank Wastes (Red Oil Safety Evaluation), LAUR-93-2809, Rev. 1 (1993).

7. R. M. Wagner, Investigation of Explosive Characteristics of PUREX Solvent Decomposition Products (Red Oil), HW-27492, March 17, 1953.

8. G. S. Nichols, "Decomposition of the Tributyl Phosphate - Nitrate Complexes", DP-526, November, 1960.

9. H. D. Harmon, M. L. Hyder, B. Tiffany, L. W. Gray, and P. A. Soltys, "Behavior of Tributyl Phosphate in A-Line Processes", DP-1418, August, 1976.

10. Letter, R. J. Gordon to S. T. Augsberger, "Environmental Testing-Explosion Hazards Tributylphosphate (TBP) / Nitrate Stability", October 9, 1984.

11. Letter, R. J. Gordon to S. T. Augsberger, "Environmental Testing-Explosion Hazards Tributylphosphate (TBP)/ Nitrate Stability", May 15, 1985.

12. M. C. Thompson and S. Cavin, private communication, March, 1994. 
13. M. V. Vladimirova, I. A. Kulikov, and A. A. Kuprii, "Thermal Oxidation and Nitration of Systems of Tri-n-Butyl Phosphate and Diluent, Atomnaya Energiya, 71, 4, 337 (October 1991), English translation by Plenum Publishing Co., 1992, p. 838.

14. (a) P. Wilcox, BNFL, "Report on Tomsk-7 Accident", citing information provided by A. Dimitriev, Head of Fuel Cycle Plant Inspectorate, Russian Gosatomnadzor, May 12, 1993. (b) "About the Causes and Effects of the Accident in Tomsk-7, Information-Analytical Investigations", Unpublished report of a Japanese investigating team, 1993.

15. W. Davis, Jr., J. Mrochek, and C. J. Hardy, "The System: Tri-n-Butyl Phosphate (TBP)-Nitric Acid-Water-I Activities of TBP in Equilibrium with Aqueous Nitric Acid and Partial Molar Volumes of the Three Components in the TBP Phase", J. Inorg. Nucl. Chem, 28, 2001 (1966).

16. F. Albert Cotton and Geoffrey Wilkinson, Advanced Inorganic Chemistry, Fourth Edition, John Wiley and Sons, New York, 1980, p. 479.

17. Joint United States/Russian Federation Meeting on Radiochemical Processing Safety, Richland, Washington, September 24-25, 1993, U. S. Dept. of Energy Report, 1993.

18. W. W. Schulz and J. R. Navratil, Science and Technology of Tributyl Phosphate, Vol. I, CRC Press, Inc., Boca Raton, FL, 1984.

19. A. J. Moffat and R. D. Thompson, "The Chemical Stability of Tributyl Phosphate in Some Nitrate and Chloride Systems", J. Inorg. Nucl. Chem. 16, 365 (1961).

20. J. R. Smith, Savannah River Technical Center, Private Communication, June, 1994.

21. G. S. Barney and T. D. Cooper, The Chemistry of Tributyl Phosphate at Elevated Temperatures in the Plutonium Finishing Plant Process Vessels, WHC-EP0737, March 11, 1994.

22. M. L. Hyder, Safe Conditions for Contacting Nitric Acid or Nitrates with Tri-n-Butyl Phosphate (TBP), WSRC-TR-94-059, January 31, 1994.

23. M. L. Hyder, "Best Estimate Calculation for Oxidation of TBP by Nitrate", SRTEAG-940093, May 2, 1994.

24. N. J. James and G. T. Sheppard, "Red Oil Hazards in Nuclear Fuel Reprocessing", Nuclear Eng. \& Design 130, 59 (1991). 
WSRC-TR-94-0372

Rev. 0

. Page 15 of 18

25. Fauske and Associates, Tributyl Phosphate-Nitric Acid Reaction and Vent Requirement, FAI/94-68, July, 1994. 
WSRC-TR-94-0372

Rev. 0

Page 16 of 18

Figure 1: TBP Acid Hydrolysis \& Reaction Rates

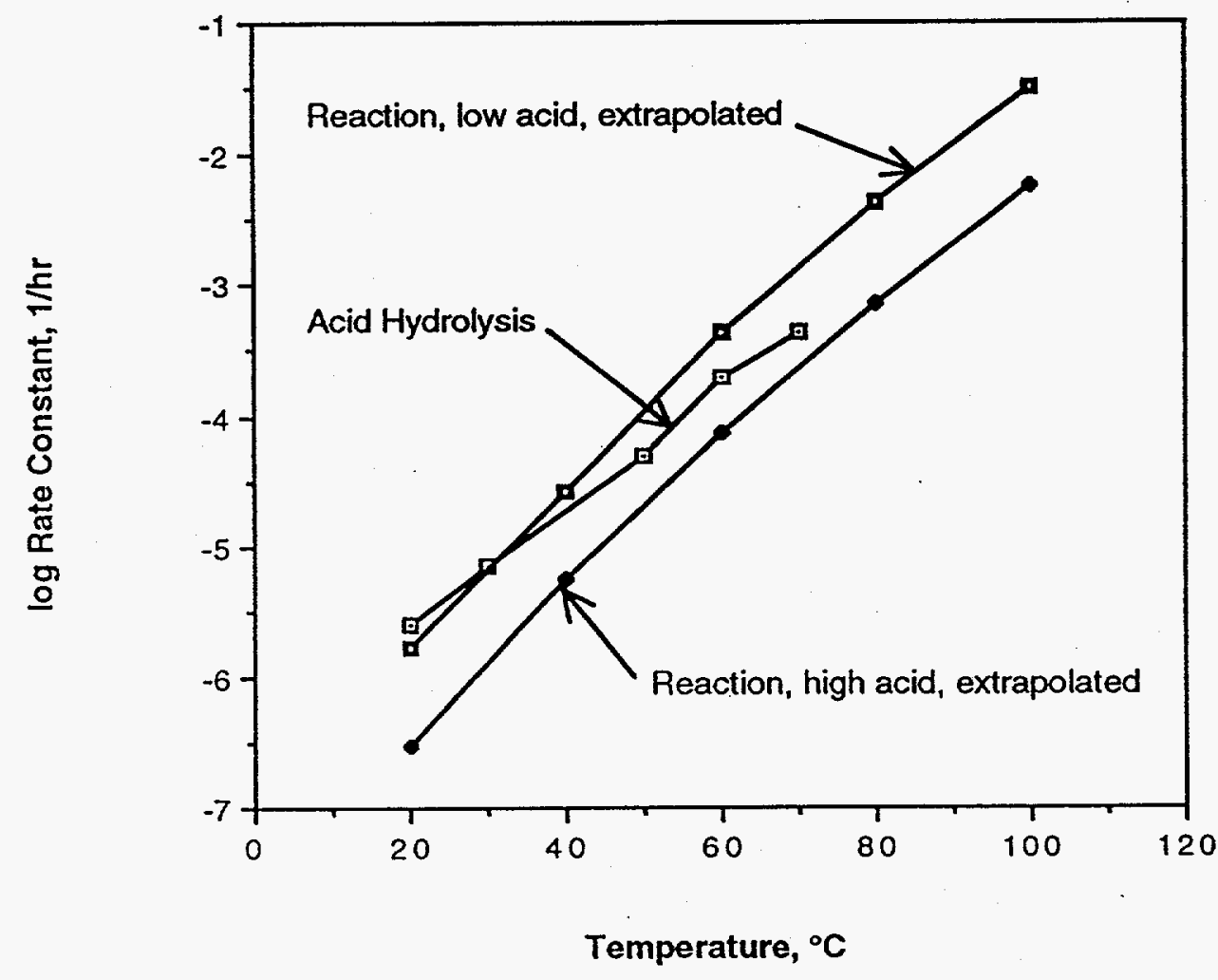


WSRC-TR-94-00372

Rev. 0

Page 17 of 18

Figure 2: Butene Production vs. Temperature

Temperature, ${ }^{\circ} \mathrm{C}$

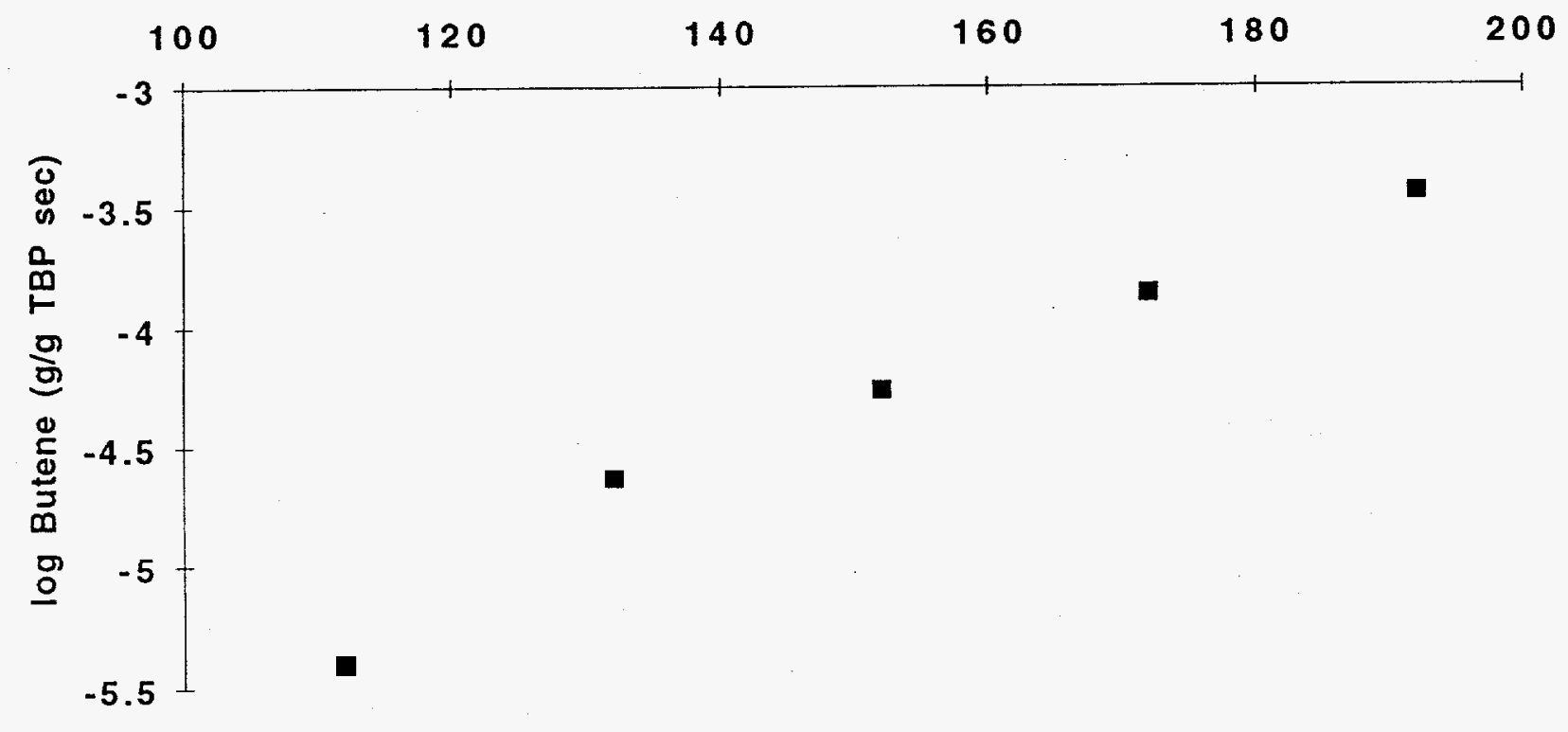


Figure 3: Effect of Vent Size on Internal Pressure

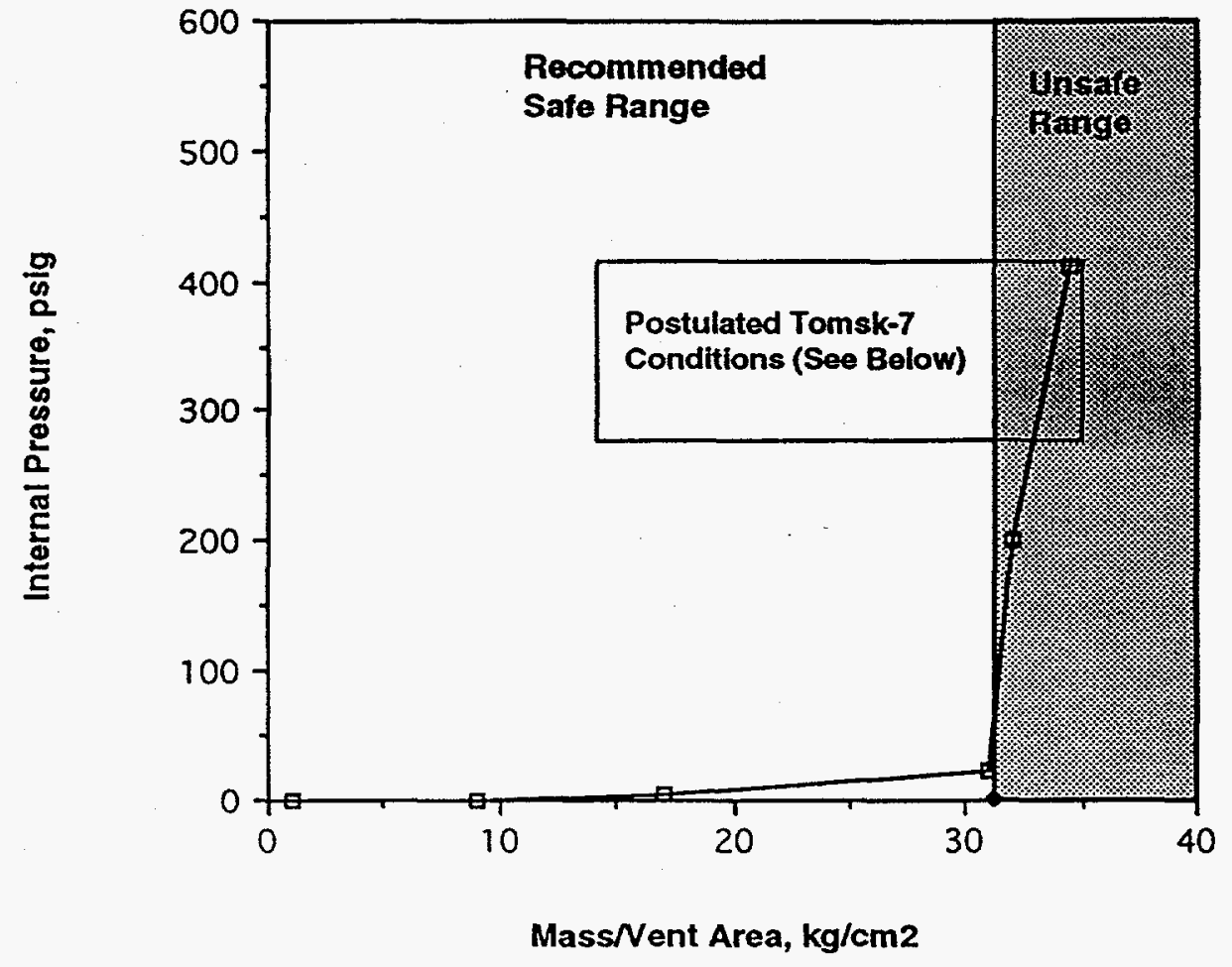

Notes:

1. Data in curve are from Fauske (Ref. 25) or Thompson (Ref. 12).

2. Box of postulated Tomsk-7 conditions based on 200 to 500 liters organic (Refs. 1, 17) and a density of 1.35 (Ref. 15) neglecting the effect of uranyl nitrate. The vertical range of this box is an estimate of tank failure pressure beginning at about $20 \mathrm{~atm}$. 